\title{
DESIGN AND TESTING OF A CALORIMETER FOR MICROBIOLOGICAL USES
}

\author{
TERUYUKI FUJITA, KAZUKO NUNOMURA, IKUZO KAGAMI, \\ AND YOSHIKAZU NISHIKAWA*
}

The Institute of Applied Microbiology, University of Tokyo, Tokyo, and *Rheska, Co., Ltd., Tokyo

(Received August 11, 1975)

\begin{abstract}
A rotatory calorimeter of the conduction type was remodelled to fit the continuous study of microbiological processes. Provisions for aeration and agitation made it possible to be used for calorimetric studies of microorganisms aerobically growing in a 5-ml culture. The time constant of the calorimeter response was found to be $8 \mathrm{~min}$ and the calorimeter could be used for most kinetic studies on microbial cultures.

By using a separate culture apparatus, quantitative analyses can be made on samples taken from parallel culture and the results can be correlated with the thermogram obtained with the calorimeter. The performance of the instrument was demonstrated with the aerobic growth of Saccharomyces cerevisiae. Calorimetric study by the use of the present model may give a detailed information at the cellular level about microbial growth processes when combined with turbidity measurements.
\end{abstract}

Calorimetry of microbial metabolism was used in the past mainly in the study of microbial energetics to show the degree of utilization of nutritional substances $(1,2)$. With the development of modern instruments, it has been shown that, both in growing and non-growing microbes, the processes involved can be studied quantitatively by calorimetry (3). Most microbiological studies are, however, restricted so far to anaerobic processes. This is because the calorimetric operation of aerobic processes is technically more difficult than that of anaerobic ones. The former requires forced aeration which is usually accompanied to some extent with thermal disturbances. Although several calorimeters commercially available have been used in microbial studies $(4,5)$, each of them has its own disadvantage inherent in its design. Among these, the instruments used by ERIKSSON and WADSÖ (6) appears most suitable for aerobic growth experiments, but it seems inconvenient for experiments with a small volume of samples. Requirements 
specific for microbial uses are as follows: (1) An instrument should retain its operation stability for a long period, (2) the culture vessel can be repeatedly sterilized, (3) there should be some provision for agitation and aeration, (4) the time constant of the instrument response should be small for kinetic measurements, and (5) a sampling device or an apparatus for parallel culture is desirable. Design and testing of a calorimeter modified to meet these conditions will be described in this paper.

\section{EXPERIMENT}

Calorimeter. The calorimeter used in this study is a modified version of the rotation microcalorimeter originally designed by AMAYA et al. (7). The principal arrangement of the calorimeter is shown in Fig. 1. An aluminum block serving

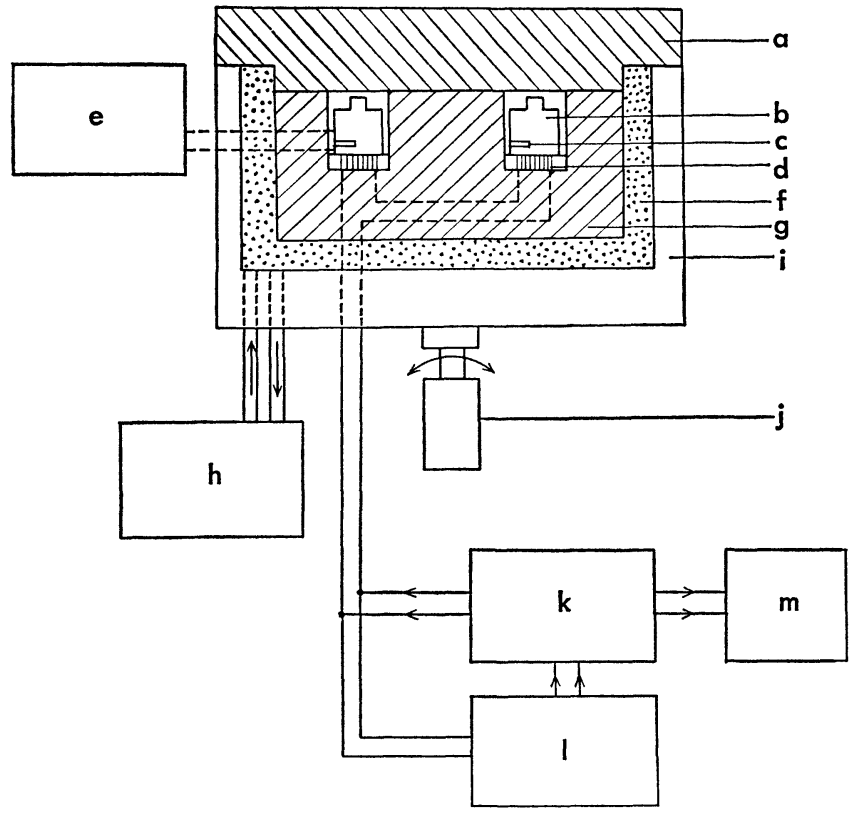

Fig. 1. General view of the calorimeter.

a, lid; b, reaction vessel; c, calibration heater; $d$, thermopile plate; e, heater power supply; $\mathrm{f}$, thermostatic bath; $\mathrm{g}$, heat sink; $\mathrm{h}$, thermostat controller; $\mathrm{i}$, thermal insulation; $\mathrm{j}$, rotating device; $\mathrm{k}$, compensation circuit; 1 , differential amplifier; $\mathrm{m}$, recorder.

The rotation axis $(\mathrm{j})$ is placed horizontally and the main part of the calorimeter containing two reaction vessels rotates about this axis alternately in clockwise and anticlockwise way. Consequently, the cylindrical axes of the reaction vessels are kept in parallel with the horizontal rotation axis.

as a heat sink contains two identical reaction vessels, one for active and the other for reference uses. The temperature of the heat sink is regulated within $\pm 0.02^{\circ}$ 
by circulating thermostated water. Thermopile plates situated between the vessels and the heat sink are used for temperature indication and compensation of the heat effect produced in the active vessel. The thermopile plate used is a semiconductor thermoelectric module with a temperature sensitivity of $6.5 \mathrm{mV} /{ }^{\circ} \mathrm{C}$ and serves also as a path through which heat passes from the reaction vessels to the sink or vice versa. Initiation of the reaction and stirring of the reactants are effected by rotation of the calorimeter using a reversible motor at $4 \mathrm{rpm}, 360^{\circ}$ clockwise and anticlockwise, alternately. The heat effect produced in the active vessel is compensated by the Joule heating or the Peltier cooling using the active thermopile plate according to the process taking place in the vessel in such a way as to make zero the temperature difference between the two vessels. The quantity of the electrical energy supplied to maintain the balance is continuously recorded as the calorimeter signal which is proportional to the rate of the heat flow passing through the thermopile of the active reaction vessel. In the range of the highest sensitivity, the calorimeter gives $30 \mathrm{~mm}$ deflection of the recorder pen for a heat flow of $1 \mathrm{cal} / \mathrm{hr}$. The heat quantity evolved is proportional to the chart area under the thermogram. Since the instrument was originally designed as a generalpurpose calorimeter, several modifications to the standard design was made to meet the conditions described above as necessary for microbial uses. The reaction vessels were substituted by culture vessels with provisions for aeration and a separate culture apparatus as described below.

Culture system. The culture system consists of three parts; (a) a culture vessel, (b) an aeration assembly, and (c) a culture apparatus for sampling uses.

The culture vessel shown in Fig. 2 is a short-necked cylindrical flask made of Pyrex glass with a diameter of $50 \mathrm{~mm}$ and a height of $30 \mathrm{~mm}$. The vessel has an

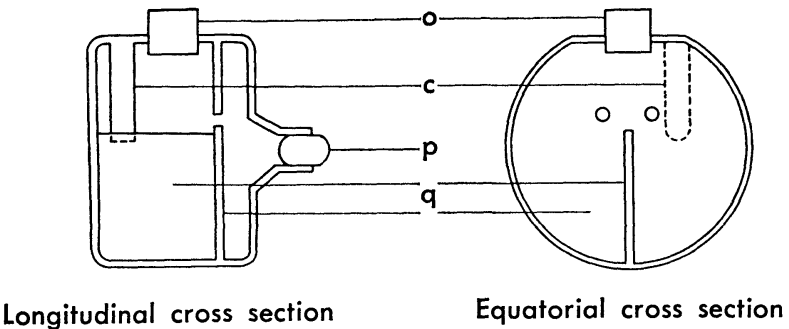

Fig. 2. Longitudinal and equatorial cross sections of a culture vessel.

$o$, Silicone rubber cap; c, calibration heater; $p$, cotton-wool plug; $q$, partition walls.

opening, two partition walls, and a calibration heater contained in a glass tube. The opening on the wall is covered by a Silicone rubber cap and serves for cleaning. The perforated partition wall in parallel with the bottom prevents the cotton-wool plug from wetting. Another partition wall vertical to the bottom separates the reactants prior to operation and serves as a baffle plate during the measurement. 
The culture vessel with a capacity of $40 \mathrm{ml}$ is usually charged with $5 \mathrm{ml}$ of liquid in order to secure efficient aeration and agitation.

The aeration assembly is shown schematically in Fig. 3. The original aim to obtain a stable base-line in the thermogram by using a controlled rate of air flow

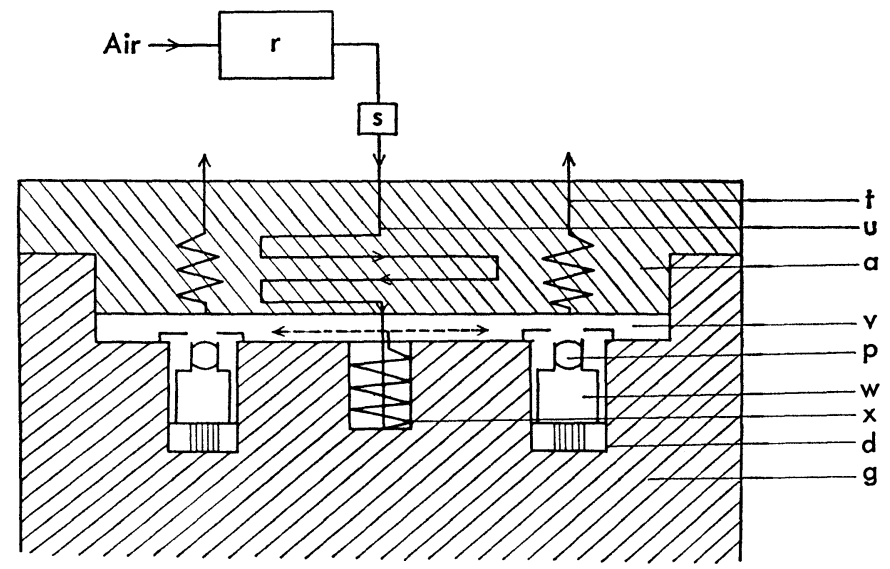

Fig. 3. Aeration assembly.

$r$, peristaltic pump; $s$, sterilizing filter; $t$, outlet copper tube; $u$, inlet copper tube;

a, lid; v, air reservoir; $p$, cotton-wool plug; w, culture vessel; $x$, heat exchange unit; $\mathrm{d}$, thermopile plate; $\mathrm{g}$, heat sink.

was not achieved with any piping methods, because the heat effect of water vaporization was not completely cancelled out. In the present design, moist air is pumped in at a flow rate of $15 \mathrm{ml} / \mathrm{min}$ by a peristaltic pump via a sterilizing filter (Swinex-13 Filter Unit, Millipore Filter Corp., U.S.A.) to an inlet copper tube in the lid and then to the heat exchange unit which consists of spiral copper tubings with an internal diameter of $2 \mathrm{~mm}$ and a total length of $100 \mathrm{~mm}$ immersed in Silicone grease filled in a hole of the heat sink for thermal equilibration. After passing through the heat exchange unit, the air fills the space between the lid and the heat sink and enters the culture vessels via the cotton-wool plugs. The space serves as an air reservoir with dimensions of $200 \times 140 \times 15 \mathrm{~mm}$. Excess air and exhaust gas are allowed to flow out through the outlet copper tubes in the lid. Silicone rubber tubes with an internal diameter of $3 \mathrm{~mm}$ were used as connections between the copper tubes, the pump, and the heat exchange unit. By using the present aeration system, the thermal disturbance possibly caused by aeration could be made negligibly small.

Since it is difficult to take out samples from the culture vessel without any thermal disturbance during the course of calorimeter measurement, parallel culture for the sampling use is made using a separate thermostated culture apparatus. The culture apparatus consists of a drum made of Styrofoam with a diameter 
of $200 \mathrm{~mm}$ and a thickness of $60 \mathrm{~mm}$ which holds two calorimetric culture vessels and is rotated through a geared motor at $4 \mathrm{rpm}$.

Cultures are carried out separately in a thermostated chamber and provide sample materials produced under the same condition as in the calorimeter for biological and chemical analyses.

Organism and culture medium. The yeast studied (Saccharomyces cerevisiae) was a parent strain of a respiratory-deficient mutant kindly provided by Dr. T. Gunge, Mitsubishi Chemical Industries Research Laboratory. Kilkenny and Hinshelwood's synthetic medium (8) was used as a growth medium.

Operational procedure. The active culture vessel was charged with $4.5 \mathrm{ml}$ of the medium and $0.5 \mathrm{ml}$ of the inoculum, each in a separate compartment, by means of a syringe. The reference vessel was similarly charged with the medium and a buffer solution instead of the inoculum used in the active vessel.

These procedures were carried out under sterilized conditions and then vessels were placed in position in the calorimeter. After temperature equilibration, the duration of which was $3 \mathrm{hr}$, the calorimetric measurement was started by rotating the calorimeter. The inoculum previously separated by the partition walls was mixed with the growth medium by starting the rotation and microbial growth was initiated in the active culture vessel, while the medium and the buffer solution were similarly mixed together in the reference vessel. The calorimeter was continuously rotated for aeration and agitation during the culture.

Chemical analysis. Glucose was determined enzymically using the Glucostat method and ethanol was determined by gas chromatography. Turbidity of the culture was measured at $550 \mathrm{~nm}$ with a Shimadzu Spectronic 20 spectrophotometer by using $0.1-\mathrm{ml}$ microcuvettes.

\section{RESULTS AND DISCUSSION}

\section{Electrical calibration}

The electrical calibration data were obtained as shown in Fig. 4 by electrically heating at a known rate for different periods with concomitant rotation of the calorimeter. The experiments were made with the culture vessels charged with $5 \mathrm{ml}$ of distilled water. Results show that the thermal lag of the instrument affects the time course of the record. The rising part of the recorded curves is approximately exponential with a time constant of $8 \mathrm{~min}$. As shown in Fig. 5 the relationship between the chart area under the calorimetric records and the heat input is linear, as theoretically expected for a system of the first-order response. Since the transient periods of microbiological processes are usually long compared with the time constant of the calorimeter, the present calorimeter not only gives the total quantity of heat produced in the active vessel but can also be used for the kinetic study of microbiological processes. 


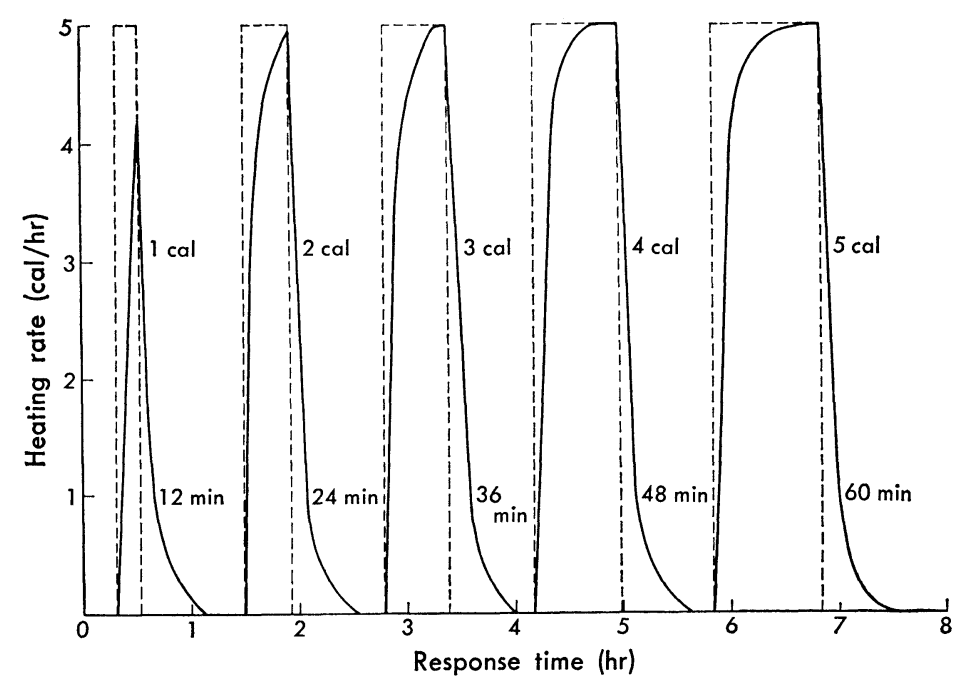
periods.

Fig. 4. Calorimetric records given by a constant power heating for different

- observed curve; ----- heat input.

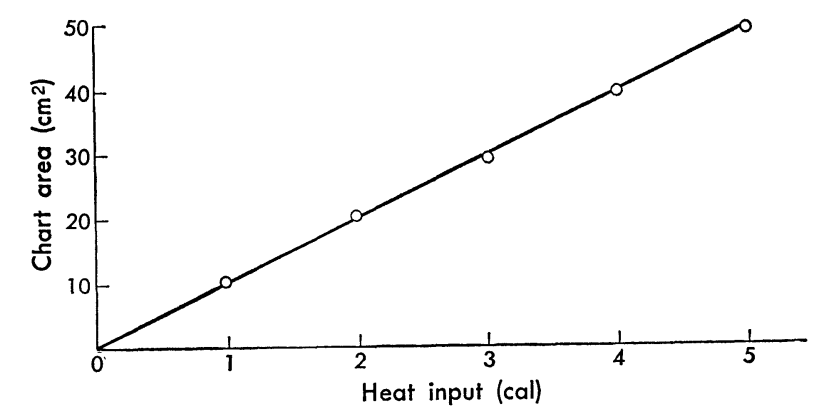

Fig. 5. Relationship between the chart area and the heat input.

\section{Test of growth conditions}

As the agitation and aeration may limit the growth rate in the calorimeter, the present model was tested for its efficiency in the aerobic microbial growth using yeast as a test organism. Figure 6 shows the growth curves obtained by the use of different culture methods. The growth curve obtained with the calorimeter without taking calorimetric data is approximately the same as that with the culture apparatus described above. This graph also shows that the satisfactory growth rate can be obtained under the culture condition used by referring to several growth curves obtained with several other culture methods.

\section{Performance of the calorimeter}

The performance of the calorimeter was tested by measuring the heat evolution 


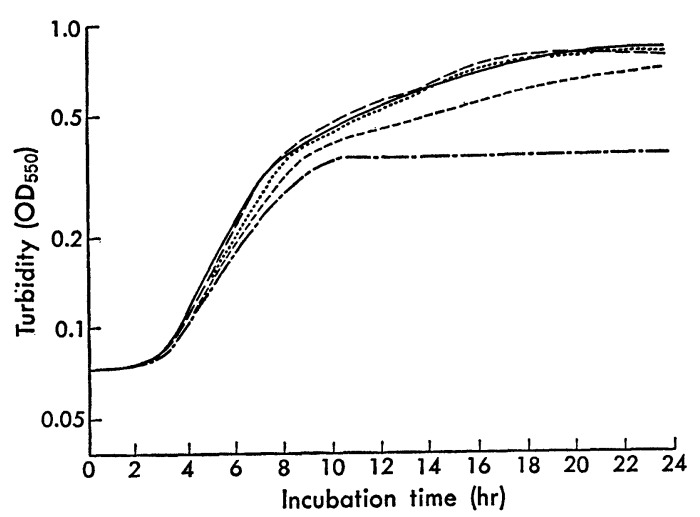

Fig. 6. Comparison of yeast growth curves obtained by different culture methods.

- - - rocking culture (20 times/min) in a $20-\mathrm{ml} \mathrm{L-form} \mathrm{tube} \mathrm{holding} 10 \mathrm{ml}$ of culture; --.-- reciprocal shaking culture (150 times/min) in a $20-\mathrm{ml}$ test tube holding $10 \mathrm{ml}$ of culture; .... rotating culture in the culture apparatus; — rotating culture in the calorimeter; - - - stationary culture in the calorimetric culture vessel. Each calorimetric culture vessel holds $5 \mathrm{ml}$ of culture.

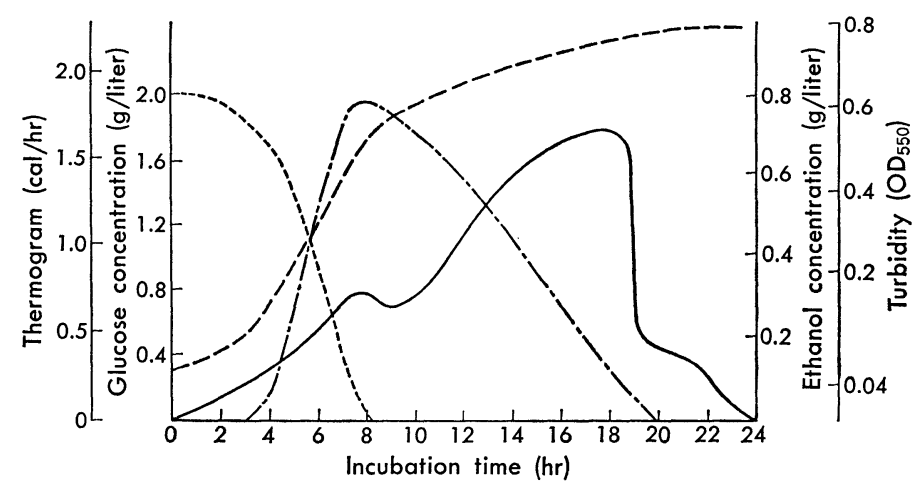

Fig. 7. Aerobic growth experiments with $S$. cerevisiae. turbidity.

thermogram; ----- glucose concentration; ---- ethanol concentration; ---

during the yeast growth. Figure 7 shows an example of the thermogram observed for the aerobic growth of $S$. cerevisiae. Turbidity, glucose concentration, and ethanol concentration found for the medium in the culture apparatus are compared with the corresponding thermogram obtained with the calorimeter. The thermogram observed was quite different from that of the anaerobic growth of $S$. cerevisiae (9). The thermogram shows several distinct phases. From the results of chemical analyses the first peak was identified as corresponding to the growth on glucose and the second peak seemed to be due to the consumption of ethanol 
formed during the glucose fermentation. The origin of the shoulder appearing after the second peak remains yet to be studied. It seems likely that the thermogram obtained with the present calorimeter provides a detailed information about microbial growing processes, when used in combination with turbidimetric measurements. Quantitative analyses of the growth thermogram will be reported in subsequent papers.

The authors are very grateful to Dr. T. Gunge, Mitsubishi Chemical Industries Research Laboratory, for the gift of yeast strains.

\section{REFERENCES}

1) W. H. Peterson and P. W. Wilson, Chem. Rev., 8, 427 (1931).

2) E. H. Battley, Physiol. Plant., 13, 192, 628, 674 (1960).

3) W. W. Forrest, In Biochemical Microcalorimetry, ed. by H. D. Brown, Academic Press, New York (1969), p. 165.

4) J. P. Belaich, J. C. Senez and M. Murgier, J. Bacteriol., 95, 1750 (1968).

5) P. MonK and I. Wadsö, Acta Chem. Scand., 22, 1842 (1968).

6) R. ERIKsson, and I. WAdsö, In First European Biophysics Congress, ed. by E. BrodA, A. LoCKer, anp H. S. LEDERER, Proceedings Vol. IV”, Wiener Medizinischen Akademie, Wien (1971), p. 319.

7) K. Amaya, S. Takagi, and S. Hagiwara, Abstracts of papers, 2nd Japanese Calorimetry Conference, Tokyo, November (1966).

8) B. C. Kilkenny and S. C. Hinshelwood, Proc. Roy. Soc. B, London, 138, 375 (1951).

9) K. Takahashi, Agr. Biol. Chem. (Tokyo), 37 (12), 2743 (1973). 